\title{
Protée
}

\section{Quand le discours se veut langage d'un sujet plutôt qu'instance indifférente de la langue}

\section{Joëlle Réthoré et Cécile Paucsik-Tourné}

Volume 26, numéro 3, 1998

Logique de l’icône

URI : https://id.erudit.org/iderudit/030524ar

DOI : https://doi.org/10.7202/030524ar

Aller au sommaire du numéro

\section{Éditeur(s)}

Département des arts et lettres - Université du Québec à Chicoutimi

ISSN

0300-3523 (imprimé)

1708-2307 (numérique)

Découvrir la revue

Citer cet article

Réthoré, J. \& Paucsik-Tourné, C. (1998). Quand le discours se veut langage d'un sujet plutôt qu'instance indifférente de la langue. Protée, 26(3), 35-44.

https://doi.org/10.7202/030524ar

\section{Résumé de l'article}

Pour justifier une telle interprétation de certaines démarches d'écriture à visée poétique, nous tenterons de montrer que ce ne sont pas tant des signes qui nous sont " donnés " à lire qu'un langage fait de " jugements perceptuels " sur la réalité, vision préconsciente favorisant le musement du lecteur et visant à modifier suffisamment ses habitudes de lecture pour qu'il remette en cause sa pensée du monde et de sa propre place dans le monde. L'accent est non

seulement porté sur la priméité de l'expression, mais le texte appelle le lecteur à oublier le fait que toute représentation verbale relève, fondamentalement, de l'univers de la tiercéité, c'est-à-dire de la loi établie par convention comme mode communicable de représentation. Des illustrations ont été choisies dans la trilogie de Dos Passos (The 42nd Parallel et The Big Money). 


\title{
QUAND LE DISCOURS SE VEUT LANGAGE D'UN SUJET PLUTÔT QU'INSTANCE INDIFFÉRENTE DE LA LANGUE
}

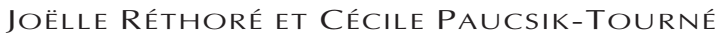

Face à l'énergie nucléaire, la lampe d'argile du poète suffira-t-elle à son propos? - Oui, si d'argile se souvient l'homme. (Saint-John Perse, 1970, 171)

L'affirmation sélective "l'équilibre appartient à l'avenir», loin d'être arbitraire, corrigerait donc un arbitraire, la trahison contingente [...] (Stengers, 1997: 80)

La très grande majorité des théories linguistiques a, avec une belle constance, retenu la langue comme son premier et longtemps unique objet, puis, devant l'échec des conséquences sociales de ses descriptions, a finalement accepté de se pencher sur la dimension du discours. Dans ses commencements, qui se voulaient avant tout scientifiques, elle n'a retenu comme pertinente que la logique du général, qui correspond, en langage peircien, à l'univers de la tiercéité. En évoluant du côté du discours, elle a accordé une place à l'existant, et ce faisant a glissé d'un univers dans un autre, celui de la secondéité, du fait, de l'occurrence. Le discours fut perçu comme instanciation de types. Ainsi la logique de l'existant fut-elle rendue dépendante de celle du général. Aujourd'hui, la théorie sémiotique inspirée des travaux de C. S. Peirce exige de penser le langage dans les termes d'un troisième univers jusqu'ici explicitement rejeté, celui du possible et de la qualité, c'est-à-dire satisfaisant aux lois de la logique du vague. En abordant à ces rivages de la priméité, elle a quitté le plan des entités discrètes, c'est-à-dire des signes, pour s'intéresser au tissu continu de la vie mentale, à sa rêverie ordinaire que Peirce appelle le musement, comme aussi à sa mémoire ancestrale des formes et des significations, en un mot le langage avec un grand $L$ peut-être, mais toujours transmis avec un petit $l$ à chacun d'entre nous, sujet plus ou moins conscient de cette filiation. La refondation récente de la linguistique historique à partir des travaux d'anthropologues et linguistes américains, pour certains datant de plus d'un demi-siècle, suscitée notamment par Ruhlen (1997) et Greenberg ${ }^{1}$, nous permet d'espérer que cette dimension fondamentale dans tous les sens du terme, que représente la priméité des phénomènes et qui est un pilier de notre humanité, se verra enfin accorder un peu d'attention de la part des linguistes et des théoriciens de la critique littéraire. 
En adoptant a priori le point de vue de la sémiotique peircienne appliquée au langage, nous faisons nécessairement une place à la notion de "ton", pour en saisir la dimension de priméité; à celle de «token", pour parler de la secondéité du signe lorsqu'il se mue en discours; et à celle de "type", lorsque l'accent est mis sur la dimension de tiercéité ou loi qui permet aux types de régir les «tokens", types qui correspondent à la norme ou encore à la langue standard.

\section{UN PEU DE PÉDAGOGIE DE LA MÉTHODE}

La richesse et l'importance du texte peircien sont telles que peu de sémioticiens exploitent les mêmes ressources offertes par ce texte gigantesque dans tous les sens du terme. Nous nous devons donc, afin de rendre nos hypothèses et notre argumentation lisibles, de mettre à plat les articulations théoriques et les notions à partir desquelles surgissent nos propres hypothèses. Nous avons retenu d'illustrer notre propos par un roman. Le phénomène déclencheur de nos inférences en relation avec le thème du numéro fut celui de l'agglutination, très présent dans la trilogie de l'écrivain américain John Dos Passos (U.S.A.), en particulier dans les "Camera Eyes», que l'on peut sans hésiter qualifier de prose poétique.

Les concepts qui nous amènent à parler de l'iconicité de la façon qui sera développée dans le cadre de cet article sont les suivants: 1) les catégories fondamentales de toute expérience que sont la priméité, la secondéité et la tiercéité; 2) la base perceptuelle de tout signe, c'est-à-dire de tout ce qui fait effectivement signe pour quelque interprète à un moment non quelconque, qui sera l'ancrage «hic et nunc", social et historique, du déclenchement de l'activité interprétative donnant lieu à de nouveaux signes interprétants du premier; 3 ) le caractère inférentiel du processus menant d'un percept à une abduction ou hypothèse de sens; 4) la dénomination de «sémiose» appliquée au processus également inférentiel, qui permet à un interprétant d'attribuer un objet à un signe; 5) la nécessaire contextualisation de l'émergence du sens à l'esprit de l'interprète; 6) la notion de «travail» associée au développement cognitif du signe originel, menant de son sens (souvent insu) à sa signification en tant que signe pour soi et pour la communauté ambiante; 7)l'importance du «point de vue» duquel a été perçu ce qui a fait signe à l'interprète, à son insu, ou sous son contrôle: la conséquence en est une variation non quelconque dans l'analyse. L'analyste doit déterminer, par exemple, si le signe est une "icône» proprement dite, ou un simple signe "iconique", deux manières de dire qui correspondent à des différences réelles du point de vue sémiotique, non pas relativement au signe lui-même mais à l'interprète. L'interprète d'une icône n'est pas tout à fait «la même personne» que celui d'un signe iconique. La différence provient d'une variation dans le jugement perceptuel ayant mené à chacune des interprétations. Autrement dit, l'analyse qui révèle que quelque chose a été une icône pour quelqu'un (son interprète) dit quelque chose d'autre que l'analyse qui conclut que le signe a été perçu comme iconique, l'adjectif révélant que l'interprète a eu conscience d'avoir affaire à un signe autre que son objet, à certains égards. Cette dégénérescence, liée à cette conscience, trahit l'imperfection du signe dans sa représentation de son objet, puisqu'il ne lui ressemble pas absolument en tous points et ne fait que partager une ou plusieurs qualités ou traits avec lui; 8) enfin, puisqu'il nous faut bien mettre un terme à cette énumération des prérequis de la lecture, la maxime pragmaticiste qui met en évidence la bonne méthode pour aboutir à la «signification " ${ }^{2}$ d'une expression pour son locuteur: ce sens est la somme des conséquences réelles imaginables et inimaginables de l'assertion du signe.

Munies de cette "boîte à outils", nous tâcherons de justifier la classification au sein de l'univers de la priméité des phénomènes qui ont retenu notre attention. Au-delà de cette classification, peu éclairante à ce stade du propos car mêlant des phénomènes assez variés, nous espérons montrer que la création en matière de langage - et pourquoi pas de langue? - surgit de cet univers bien plus sûrement que des deux autres univers, les seuls qui se soient vu 
attribuer une place dans la théorie linguistique, fûtelle pragmatique. Nous voulons aussi mettre l'accent sur l'importance du concept d'icône dans l'analyse des constituants de l'assertion ${ }^{3}$, sachant qu'une icône, au moins, est associée à chacun des rhèmes présents dans toute assertion: le motif principal de notre actuelle centration sur l'icône est le fait que si nous pouvons parler d'une "culture commune", c'est grâce au rôle qu'y jouent les icônes et non pas les symboles, dont le caractère conventionnel ne permet pas d'assumer la valeur «langagière", qualitative, imageante des échanges verbaux. L'étude du fonctionnement de la pensée interprétant une assertion met en évidence le surgissement d'icônes à l'esprit de l'interprète, avec chaque prédicat, dans tous les cas où la familiarité de l'interprète avec la variété de langue dans laquelle se passe l'échange est suffisante. Si tel n'est pas le cas, la dimension symbolique des mots vient faire écran entre lui et sa compréhension: sa conscience est alors partiellement occupée par le problème que lui pose le representamen, c'est-à-dire l'énoncé comme type symbolique.

Lorsque le signe qu'est l'assertion est familier, les icônes qui surgissent à l'esprit de l'interprète représentent la validation de sa compréhension du signe et de sa communication avec un autre, s'il est postulé que cette icône sera «la même» dans les deux esprits en train d'interpréter ce même signe.

Il peut être intéressant d'introduire la distinction entre icône et signe iconique, parce que seule la première s'impose comme "sens» à nous, indépendamment de tout contrôle immédiat, ce qui signifie que nous passons notre vie entraînés à notre corps défendant dans l'aventure de la pensée, sans la moindre distance par rapport aux signes. À cette situation, ô combien ordinaire, s'oppose un autre acte également familier, qui préscinde ${ }^{4}$ le signe de son objet dans un espace dans lequel la volonté critique du sujet a le loisir de s'exprimer, par une sorte de déclaration de sortie de l'univers de la priméité: l'accès à la tiercéité de ce travail mental peut être dû au manque de familiarité avec le signe comme à une résistance du sujet face à l'objet représenté. Seule l'enquête peut permettre de déterminer quelle est l'hypothèse la plus juste.

La grande difficulté est d'arriver à se figurer ce qu'est le "sens": est-ce une forme? Si tel est le cas, estce une image, un diagramme, une métaphore? Peirce parle le plus souvent d'image, ou encore de rêve ${ }^{5}$, c'est-à-dire d'une association imaginaire d'une occurrence (celle de l'assertion) et d'un type préalablement actualisé dans l'histoire du sujet, portant toutes les marques de cette expérience antérieure inconsciente pour la plus grande part. Comprendre ce qui nous est donné à lire ou entendre est très justement cela: laisser se libérer ce flux onirique lié à une histoire vécue dans un univers riche en couleurs et en complexité, qui est celui de cette composition permanente faite de langage, de discours et de langue, un univers réel qui marie les trois univers fondamentaux révélés par la théorie. Toutes les combinaisons sont possibles, qui multiplient les langages, les discours et les langues, pour un même individu ou pour un multiple d'individus en relation existentielle les uns avec les autres (nous hésitons à parler de "communauté», concept qui nous paraît fortement idéologique). Dans tous les cas, c'est tout un territoire d'icônes qui s'ouvre chaque fois que le sujet accède à de nouvelles langues, à de nouveaux langages, le discours articulant les deux autres.

Pourquoi ces «images» ne sont-elles pas appelées des "signes iconiques», sachant que l'image est une hypoicône, celle des trois hypoicônes qui a le plus faible degré de sémioticité, et que «hypoicône» est le concept proposé par Peirce pour renvoyer aux signes iconiques? Proposons une réponse raisonnable à cette question: c'est le processus objectivant et métasémiotique qu'est l'analyse de ces icônes - qui ont surgi dans nos esprits lors du phénomène de compréhension, c'est-à-dire lors de la sémiose - qui transforme ces icônes en des signes iconiques: cette objectivation est assurée soit par le sémioticien, soit par l'interprète lui-même. L'activité d'analyse a pour effet de modifier le statut des formes: ce qui fut une icône dans l'effet du signe sur l'esprit de l'interprète devient image ou diagramme, c'est-à-dire un signe 
iconique dans le langage du sémioticien. Pour ne pas s'y tromper, il importe de retenir que, dans le moment précis de leur émergence, c'est l'absence totale de distance entre le mot et son sens qui fait du second une icône, le mot et le sens ne faisant alors vraiment qu'un. Comment expliquer ce phénomène? Par l'intimité du mariage des trois catégories de l'expérience, qui condense en un point discret, la forme verbe, l'interprétation sémantique de la relation entre les sujets, généralement représentés dans l'assertion par des indices ${ }^{6}$. Le verbe, considéré isolément, en tant que partie du discours, est certes un symbole, en attente d'un indice (au moins) qui autorisera son assertion; par contre, ce même verbe, lorsqu'il est impliqué dans une assertion, un dicisigne, est l'élément de tiercéité qui interprète quelque chose qui n'est pas second dans son mode d'être; et le verbe, quant à lui, ne peut revendiquer comme élément de secondéité que les indices temporels et aspectuels qui lui sont affectés: fondamentalement, en tant qu'élément du répertoire lexical de la langue, le verbe est une représentation authentique et donc s'affiche comme médiation entre deux autres choses (la relation fût-elle réflexive), et donc comme tiercéité. C'est cela même qui en fait l'interprétant dans le signe, en tant que constituant de l'assertion. Mais c'est sa place au sein de la prédication qui signale son statut de verbe.

Nous constatons que, pour le moment, rien n'a encore été dit de son "sens»: or, c'est ce dernier qui le transforme en une icône dans l'esprit de l'interprète, une icône qui est censée être «la même» que celle qui avait surgi précédemment dans l'esprit du locuteur (ou scribe).

\section{DeuX CONSÉQUenCes À la DistinCtion ENTRE ICÔNE ET SIGNE ICONIQUE}

La première conséquence à prendre en compte est le rôle fortement idéologique des signes linguistiques, en raison du statut sémiotique très particulier des verbes. Transformer tous ces interprétants dans les signes (que sont les verbes) en «icônes» les met, au moins pour un temps, à l'abri de nos esprits critiques de lecteurs avisés. Ainsi, nous ne manquerons pas de constater que le procédé d'agglutination fortement représenté dans U.S.A. peut avoir été largement inconscient chez l'auteur, la version langage de la représentation l'emportant sur le souci délibéré, autocontrôlé par l'auteur, de bouleverser les règles de la représentation graphique de la langue, projet qui est cependant également présent chez lui?

La seconde conséquence est l'accès de ce langage partiellement idiosyncrasique à la dimension du discours, ce dernier étant à considérer comme assumé puisque publié: cet accès s'opère comme un coup de force de la priméité dans la secondéité, en violation apparente de la hiérarchie des catégories ${ }^{8}$, mais en harmonie avec l'ontogenèse du langage, avec certaines ambitions affichées par l'auteur «énonciateur " 9 d'accéder ultérieurement à la régularité et à la norme de la tiercéité.

En tant que langage, c'est-à-dire pur ensemble de qualités, de "tons", sa relation à ses objets ne peut que participer du même mode d'être, celui de la priméité des signes «iconiques", nécessairement "rhématiques", cette tiercéité (doublement dégénérée) révélant que la relation entre le signe et son objet est perçue en tant que telle par l'interprétant sous la forme d'une hypothèse ou abduction. L'univers tonal a pour caractéristique de tout faire baigner dans la dégénérescence des autres catégories phénoménales que sont l'icône et le rhème. Le travail considérable que représente l'élaboration de la théorie des graphes existentiels ${ }^{10}$, qui sont à penser comme les icônes mêmes du fonctionnement de la pensée, nous semble susceptible de fonder une théorie du «langage» comme priméité authentique de toute activité de représentation humaine depuis notre entrée dans la vie. En effet, ces graphes-icônes, en nous mettant "directement» 11 au contact, d'une part, de la secondéité caractéristique de la dimension d'objet, d'autre part, de la tiercéité ${ }^{12}$, masquent totalement la priméité du signe par rapport à lui-même, dans sa dimension monadique. S'il reste de la priméité dans les graphes, celle-ci semble réservée aux deux trichotomies auxquelles appartiennent icônes et 
rhèmes, la priméité du signe monadique n'étant pas représentée ni perçue «en tant que telle». Cet «oublié tonal», en quelque sorte, est le langage lui-même, fondement incontournable de toute langue (dont la priméité est faible, puisque la langue est faite de types, qui sont des troisièmes) et de tout discours (dont la priméité est également non authentique, puisque le discours est fait d'occurrences "hic et nunc", instances secondes des types dont il vient d'être question).

\section{LES TRACES DU MUSEMENT: DOS PASSOS} Le phénomène d'agglutination chez John Dos Passos

Nous n'aurions su, dans le cadre de cet article, faire l'impasse sur la démarche de John Dos Passos, car c'est elle qui suscita, il y a plusieurs années, notre interrogation sur la priméité du langage comme opposition à la tiercéité de la langue: la création, nous le savions par le sens que Peirce donne à l'abduction, ou production d'une hypothèse, trouve son terreau dans la priméité; mais le phénomène que nous avons appelé «agglutination", à savoir la soudure de deux morphèmes qui se suivent dans la chaîne de l'énoncé ${ }^{13}$, n'était pas simple création, il était désir de révolution. Certes, en ce sens, relevait-il d'abord de la tiercéité, mais il s'avère que le procédé est utilisé de façon non consistante par l'auteur: les mêmes syntagmes, ou des syntagmes proches, sont parfois agglutinés, et parfois pas. Il nous apparut que l'objet d'une telle pratique de l'écriture poussait à sa représentation de façon compulsive: «ça» parlait, ou plutôt, «ça» écrivait, parfois agglutiné, et parfois pas, sans que le scribe puisse exercer son contrôle. Le scribe, certes, était très normalement déterminé à poser sa représentation, mais sous l'effet d'une pulsion originelle très importante relevant de la priméité des phénomènes.

C'est dans les "Camera Eye», séquences autobiographiques de la trilogie de Dos Passos, U.S.A., où se déploie peut-être plus librement que dans les autres parties le musement de l'auteur, que les occurrences d'agglutination nous ont semblé révéler cette pulsion originelle.

Ces courts segments de l'œuvre suivent le chemin parcouru par l'auteur plutôt qu'ils ne retracent sa vie, depuis une enfance vécue à l'étranger, en France notamment, jusqu'à l'âge adulte de la désillusion et de l'échec subi par la condamnation et l'exécution de Sacco et Vanzetti en 1927. Le dernier "Camera Eye» se termine par la conviction que ce sont les mots qui permettront à l'individu de lutter contre l'oppression exercée par les grands systèmes politiques et économiques (cette interprétation naît en fait de la juxtaposition des derniers mots du "Camera Eye 51", "We have only words against» et du titre de la chronique de vie qui suit immédiatement ce "Camera Eye», «Power Superpower» ${ }^{14}$ ). Mais ce ne sont pas les mots, tels que la langue nous les offre, qui permettront de mener la lutte, car ils ont souffert de l'érosion des ans et ont été galvaudés par des utilisateurs peu soucieux de respecter les grands principes énoncés par les pères fondateurs de la démocratie américaine:

America our nation has been beaten by strangers who have turned our language inside out who have taken the clean words our fathers spoke and made them slimy and foul. 15

Il faut donc reconstruire ces mots:

$$
\begin{aligned}
& \text { [...] rebuild the ruined words worn slimy in the mouths of } \\
& \text { lawyers districtattorneys collegepresidents Judges without the old } \\
& \text { words the immigrants haters of oppression brought to Plymouth } \\
& \text { how can you know who are your betrayers America. } 16
\end{aligned}
$$

Voilà donc clairement énoncé le programme d'un auteur politiquement engagé, tenté par le communisme mais trop inquiet des valeurs individuelles pour devenir homme de parti, révolté par le capitalisme effréné des années 20, qui ne profite qu'à une minorité peu scrupuleuse des valeurs démocratiques et qui n'a que le langage pour toute arme. Désir de révolution affirmé, mais qui semble parfois échapper au contrôle de l'auteur lui-même. Nous en voulons pour preuve l'absence de régularité qui gouverne les occurrences des mots agglutinés. Nous trouvons ainsi aussi bien "olive oil» ${ }^{17}$ que «oliveoil» 18 . Un exemple peut-être plus frappant nous est fourni dans le "Camera Eye 26" 19 où nous trouvons, dans l'espace de deux paragraphes, les occurrences «machineguns» et «machine-guns». Le 
syntagme "adamsapple» 20 est un autre exemple de cette fluctuation, puisqu'il apparaît, dans la dernière partie narrative du dernier volume de la trilogie The Big Money, sous la forme "adam'sapple» 21.

Ceci laisse à penser que le processus d'agglutination, bien que s'intégrant dans une volonté explicite de recréation du langage, n'est pas toujours maîtrisé par l'auteur et est parfois de l'ordre de la compulsion. Ce n'est malgré tout pas toujours le cas, ainsi que le laisse suggérer le passage dans le «Camera Eye 49» qui constitue également un programme de sens:

[...] pencil scrawls in my notebook the scraps of recollection the broken halfphrases the effort to intersect word with word to dovetail clause with clause to rebuild out of mangled memories unshakably (Old Pontius Pilate) the truth. 22

Le premier «Camera Eye» nous offre l'exemple d'un mot agglutiné "grassblade»23. L'on pourrait se contenter de mettre cette écriture particulière, non attestée par les dictionnaires, sur le compte d'une orthographe fluctuante, dont l'absence de régularité alimente régulièrement les débats lors des réformes. Il est vrai que si l'on s'en tient à une approche sémantique classique, l'on ne peut guère apporter d'explication éclairante au phénomène. La sémiotique peircienne nous permet d'appréhender les termes agglutinés, non plus comme un symbole, mais comme un légisigne deux fois dégénéré dans son rapport à son objet et à son interprétant, à savoir un légisigne iconique, nécessairement rhématique. La différence entre icône et signe iconique est pertinente ici, car elle permet de séparer les deux points de vue de l'enfant qui vit l'expérience et de l'énonciateur adulte qui nous la transmet. Examinons le contexte dans lequel le mot apparaît:

[...] when you walk along the street you have to step carefully always on the cobbles so as not to step on the bright anxious grassblades easier if you hold Mother's hand walking fast you have to tread on too many grassblades the poor hurt green tongues shrink under your feet. 24

L'enfant et sa mère sont en fuite, poursuivis par des personnes adultes qui leur jettent des pierres.
L'agglutination transforme le signe en representamen, objet d'analyse pour l'énonciateur et, partant, le lecteur. En opérant cette soudure, l'énonciateur attribue un objet différent au signe ou du moins installe une barrière entre le signe et son objet. Ce qui importe, ce ne sont pas les brins d'herbe en tant que tels, mais la perception qu'en a l'enfant. Le contexte vient conforter cette idée en mettant l'accent sur l'apitoiement de l'enfant sur les herbes foulées au pied, sorte de transfert de sa propre expérience. L'agglutination permet également de comprendre comment l'enfant découpe le réel, de façon subjective, en bloc. C'est un regard neuf qu'il porte sur le monde, non encore déterminé par les relations arbitraires des mots entre eux.

Un exemple plus significatif d'agglutination surgit dans le "Camera Eye 3", qui nous permet de saisir pleinement la priméité du langage dans lequel baigne l'enfant:

[...] and She was telling a story about Longago

Beforetheworldsfair Beforeyouwereborn and they went to Mexico on a private car on the new international line and the men shot antelope off the back of the train and big rabbits jackasses they called them and once one night Longago Beforetheworldsfair Beforeyouwereborn one night Mother was so frightened. 25

La suite agglutinée "Longago Beforetheworldsfair Beforeyouwereborn" rend compte, non pas simplement du discours que la mère tient à l'enfant que l'auteur aurait pu transcrire par le recours aux symboles donnés tels quels par la langue «long ago before the world's fair before you were born" -, mais de la perception qu'a l'enfant de ce discours. L'agglutination déconstruit les règles générales de la tiercéité, incapables désormais de présider à l'organisation des occurrences dans le discours. Ce ne sont pas des symboles que perçoit l'enfant, mais un enchainement de syntagmes incorporés les uns aux autres, autrement dit, un rythme. Le discours de la mère plonge l'enfant dans un passé qu'il n'a pas connu, et qui prend une coloration mythique. Les mots sont alors des icônes de ce monde révolu, au sens où ils tiennent lieu dans l'esprit de l'enfant de ce 
monde-là. Il s'agit toutefois de mesurer là encore la distance qui sépare l'enfant, qui a perçu le discours et qui l'a perçu comme une icône, et l'adulte qui tente de retranscrire les perceptions de l'enfant et nous livre de ce fait, non pas une icône, mais un signe iconique.

Que l'analyse sémantique traditionnelle soit impuissante à rendre compte des phénomènes d'agglutination est illustrée par l'occultation massive de ce phénomène par les critiques. Rares sont ceux qui la mentionnent seulement. Quant à ceux qui s'y sont risqués, ils ne parviennent pas à fournir une analyse très convaincante. John Brantley, par exemple, parle de «run-together words»:

There are times when the run-together words used by Dos Passos are more mannerism than elements of style, but in U.S.A. that technique is used to express patterns of speech, as in "before the worldsfair Beforeyouwereborn", of habits of mind and therefore an aid in characterization, as in Eveline's "Yourfather mustn't be disturbed, and Dearmother's [...]» or of the machine-like quality of an action as in the passage from Henry Ford's biography: "reach under, adjust washer, screw down bolt, shove in cotterpin, reachunderadjustwasher, screwdown bolt, reachunderadjust screwdownreachunderadjust » [...] 26

Quelques remarques sur ce commentaire succinct s'avèrent nécessaires. Tout d'abord, l'auteur de l'ouvrage n'a pas jugé utile de mener une véritable enquête sur ce qu'il appelle les "run-together words". Il s'agit, selon lui, d'une technique, mais il ne la nomme pas puisqu'il se contente de qualifier un nom («words»). Qu'entend-il ensuite par «more mannerism than elements of style»? Soulignons enfin qu'il ne mentionne pas tous ces mots agglutinés qu'on rencontre au fil des pages tout au long de l'œuvre et qu'on ne peut expliquer ainsi. Peut-être est-ce ce phénomène qu'il range sous l'étiquette "mannerism»? Si tel est le cas, il nous semble irrecevable d'en minimiser la fréquence, comme il le fait avec le présentatif «there are times».

L'interprétation qu'il donne des longs segments agglutinés témoigne d'une confusion entre l'icône et le signe iconique. L'exemple suivant permettra d'illustrer cette différence:
[...] his voice was in Minnesota but

dontjaunerstandafeelesgottogetup I got a date

animportantengagementtoseeabout those lots ought

nevertohavestayedinbedsolate [...] Cant you letafellerbe? You're

in cahoots withem thaswhassematteris I know

theyreouttorookme they think Imagoddamsucker

tomadethatdeposit I'll showem Illknockyergoddamblockoff. 27

L'opacité d'une telle séquence ne permet pas de la poser comme icône de l'idiolecte du personnageénonciateur. Plus qu'elle ne révèle une caractéristique de ce personnage, il nous semble qu'elle apporte une indication sur le jugement perceptuel de l'auteurénonciateur qui, encore une fois, saisit le monde comme un tout, qu'il structure à sa manière, emporté par le flux continu de son musement et ne laissant affleurer que certaines abductions.

Si les premiers «Camera Eye» tentent de saisir les sensations de l'enfant à travers ses propres perceptions, en revanche les derniers témoignent de l'importance qu'a prise l'agglutination dans la perception du monde. Par l'agglutination, en effet, l'auteur tente de recapturer la vision neuve de l'enfant et fait de l'agglutination un instrument de rénovation, voire de révolution du langage et de la société.

L'auteur-narrateur s'associe à l'Amérique vaincue ( We stand defeated America» ${ }^{28}$ ), mais ce constat d'échec n'est pas total, puisqu'il coïncide avec le début de la lutte par les mots. La fin de l'œuvre et donc, du travail de l'écrivain, considéré par Dos Passos comme une sorte d'historien de second ordre, rendue explicite dans le "Camera Eye 50" ("our work is over the scribbled phrases the nights typing releases the smell of the printshop the sharp reek of newprinted leaflets the rush for Western Union stringing words into wires the search for stinging words to make you feel who are your oppressors America " ${ }^{29}$ ), marque un nouveau départ initié par les condamnés à mort Sacco et Vanzetti:

[...] but do they know that the old words of the immigrants are being renewed in blood and agony tonight do they know that the old American speech of the haters of oppression is new tonight in the mouth of an old woman from Pittsburgh of a husky 
boilermaker from Frisco who hopped freights clear from the Coast to come here in the mouth of a Back Bay socialworker in the mouth of an Italian printer of a hobo from Arkansasthe language of the beaten nation is not forgotten in our ears tonight the men in the deathhouse made the old words new before they died. 30

Toutefois, ainsi que nous l'avons déjà fait remarquer, les occurrences d'agglutination sont totalement irrégulières, ce qui donne à penser que l'auteur-énonciateur n'a pas toujours conscience de ce phénomène. C'est ainsi que des soudures inattendues surgissent dans le fil du discours comme autant de points d'émergence de la priméité dans la secondéité de l'écriture: «at the head of the valley in the dark of the hills on the broken floor of a lurchedover cabin a man halfsits halflies" 31 , ou bien:

[...] the position offered the opportunity presented the collarbutton digging into the adamsapple while a wooden image croaks down a table at two rows of freshlypressed gentlemen who wear fashionably their tailored names. 32

Le «Longago Beforetheworldsfair

Beforeyouwereborn", loin d'être un simple jeu de l'auteur sur le langage, une fantaisie d'écrivain, nous semble plutôt éclairer les abductions opérées par l'auteur lorsqu'il n'était encore qu'un enfant, témoignant ainsi de l'élaboration de son expérience collatérale, expérience dont l'auteur n'a pas pris pleinement la mesure. C'est ainsi qu'il ne s'est jamais fait l'interprète de cette idiosyncrasie. Le contraire eût été étonnant. L'on ne peut en permanence analyser ce qui se construit en nous. Certes, Dos Passos théorisera la fonction de l'écrivain dans certains articles, insistant sur le rôle du langage dans le renouvellement de la société mais, nulle part, il ne parlera de l'agglutination dont il ne sera jamais que le scribe. C'est bien que celle-ci puise ses racines dans l'univers de la priméité, dans le musement de l'auteur, univers peu partageable car il est la base de notre identité et de notre intimité. Peut-être est-ce pour cela que ce phénomène d'écriture a été occulté par les critiques et les traducteurs qui ne le mentionnent même pas en note?
L'agglutination apparait dans les autres parties de la trilogie, à l'exception des «Newsreels» qui la rendent impossible de par leur nature même. L'auteur, dans ces sections, ne parle pas mais laisse s'exprimer la voix des autres à travers coupures de journaux, bribes de chansons populaires, dictons, slogans. La présence de l'agglutination dans les parties narratives témoigne de la nature compulsive de ce type d'écriture puisqu'elle trahit la présence de l'auteur, alors qu'il manifeste par ailleurs la volonté de s'effacer et de laisser parler les personnages. U.S.A., ainsi qu'il l'annonce dès l'ouverture, est d'abord "the speech of the people». La trilogie est très fortement caractérisée par l'absence d'intériorité des personnages, qui parlent mais ne pensent ou ne sentent pas. Malgré l'utilisation massive du discours indirect libre, rares sont les moments où l'auteur nous laisse pénétrer à l'intérieur des personnages pour découvrir de quels sentiments ou raisonnements ils sont nourris. L'agglutination semble venir combler le manque de chair et de corps des personnages, un peu à l'insu de son auteur, un peu comme si ce qu'il cherchait à refouler, la vie, constamment tenue en échec dans toute l'œuvre, resurgissait par ailleurs, par le langage et la matérialité même de l'écriture.

Parce qu'il a déstabilisé le statut symbolique des mots en faisant basculer les légisignes vers une valeur iconique qui lui est personnelle, Dos Passos a couru le risque de ne pas être compris. L'on est en droit de se demander, par exemple, si, outre les problèmes que soulève toute traduction en général, le public français a pu réellement avoir accès à l'œuvre de Dos Passos.

\section{CONCLUSION}

Le propre du langage semble être l'affirmation de la nécessité peut-être moins d'interrompre le temps que de remettre en cause la dimension de temps propre au discours. Nous pourrions aussi considérer le langage soit comme re-traduction d'un «tempscontinu", après une première "traduction" en languediscours, soit comme retour au temps-continu, un temps compatible avec la dislocation des entités. D'où des effets - pures apparences - de contradiction 
interne, d'anomalie, d'entropie, rompant sans vergogne avec les usages du «temps-conçu-par-et-dansle-discours", c'est-à-dire du temps perçu, discret, chronologisé.

La démarche poétique pose, avec chaque instance, l'ontogenèse d'un discours si neuf qu'il n'est même pas encore écrit, chacune de ces instances étant un coup de force de la priméité de la pulsion poétique vers la secondéité de l'écriture et la tiercéité de l'interprétation. Le créateur force l'interprète à ouvrir les yeux sur l'immensité des significations liées à la priméité de l'univers, se refusant à participer à notre enfermement dans l'application stricte et rigide des seules règles de la langue, de sa syntaxe comme de sa sémantique. Il exige de nous que nous quittions le confort apparent de l'unité de la langue pour réellement "regarder» le chaos, pour rejouer tous les rapports entre eux des objets du monde, repenser leurs corrélations, et tenter d'y trouver notre propre place d'énonciateur, scribe et interprète, mais avant tout d'énonciateur-origine, de museur, d'être humain plein. L'instance poétique à tout moment est l'effet papillon qui perturbe le faux ordre syntaxique et sémantique, qui montre les limites du discours et récuse qu'il ait le pouvoir d'atteindre un équilibre dont le démiurge sait qu'il n'est accessible que dans le futur. L'idée d'un point d'équilibre de notre représentation du monde (dans le discours scientifique ou ordinaire) semble correspondre assez justement à l'interprétant final peircien que serait la Vérité, le but de toute recherche à l'horizon du temps. Mais il semble que l'écriture ne se satisfasse point de chercher à accéder à un état de stabilité, fût-il futur, son ambition au contraire étant de déplacer constamment ce point imaginé, de l'obliger à poursuivre une évolution qui n'aurait de chance de s'achever qu'avec la fin de tout langage et de toute pensée.

\section{NOTES}

1. Les hypothèses du linguiste américain Greenberg sont largement défendues dans l'ouvrage de M. Ruhlen: l'un comme l'autre défendent l'idée d'une origine commune de toutes les langues. L'idée est reprise dans l'excellent article de Dennis Philps sur la forme <sn> en anglais (1997).

2. Au sens que nous lui donnons dans Réthoré, Protée, 1998a.

3. Cf. Réthoré, 1989, vol. 1-4, en particulier 1.2.: «La théorie

peircienne de l'assertion", p. 618-620.

4. Sur la question de la "préscission", voir en particulier Peirce, 2.428 .

5. Cf. Peirce, 3.459: "A verb by itself signifies a mere dream, an imagination unattached to any particular occasion. It calls up in the mind an icon".

6. Ou parfois par des symboles, dans les propositions universelles de type "Tout homme est mortel».

7. Cf. ci-après la section III.

8. Selon cette hiérarchie, la tiercéité présuppose la secondéité, laquelle présuppose à son tour la priméité, l'inverse n'étant pas vrai. Les dix classes de signes obtenues par Peirce à partir de la triade authentique (Representamen - objet dynamique - interprétant final) manifestent les contraintes de cette hiérarchie, sans laquelle ces classes seraient plus nombreuses mais aussi sans signification du point de vue logique. Pour prendre un exemple, un symbole (troisième) ne peut provenir que d'un signe qui soit une règle, une habitude, une loi, ce que Peirce appelle un «légisigne " (également troisième). Tandis qu'un signe perçu comme iconique de son objet (degré le plus faible de sémioticité) peut être soit un qualisigne (premier), soit un sinsigne (second), soit un légisigne (troisième), ce qui revient à dire que n'importe quel signe envisagé dans sa "talité» peut être, peu ou prou, iconique de son objet. Un qualisigne, cependant, ne pourra être ni indiciaire, ni encore moins symbolique de son objet. Voir G. Deledalle, 1979.

9. Cf. J. Réthoré, «L’Entour du signe écrit: énonciation et lecture. De la nécessaire division du sujet ", Degrés, à paraître. L'énonciateur y est posé comme se livrant au musement jusqu'au moment où une compulsion réelle le pousse à passer à l'acte de locution ou écriture, ce passage pouvant être assuré par une autre personne, dans le cas du discours rapporté par exemple.

10. Cf. Peirce, vol.4, notamment, pour la présentation de sa théorie; ainsi que les nombreux travaux multigraphiés de M. Balat.

11. C'est cela qui en fait des icônes au sens strict, et non de simples signes iconiques.

12. Rhèmes et indices construisent des propositions, c'est-à-dire des dicisignes, lesquels sont organisés en arguments.

13. "Agglutination: processus de soudure de deux mots", L. Tesnière, $1976: 666$

14. The Big Money, "Camera Eye 51 », p. 1155.

15. Ibid., "Camera Eye 50 », p. 1105.

16. Ibid., "Camera Eye 49», p. 1084.

17. Nineteen Nineteen, "Camera Eye 28", p. 351.

18. Ibid., "Camera Eye 33 ", p. 461.

19. The 42nd Parallel, "Camera Eye 26", p. 290-291.

20. Nineteen Nineteen, "Camera Eye 37 ", p. 544, "Camera Eye 44 ", p. 755 .

21. The Big Money, "Mary French", p. 1176.

22. Ibid., "Camera Eye 49", p. 1083.

23. The 42 nd Parallel, "Camera Eye $1 "$, p. 21. 
24. Les espacements correspondent à la typographie adoptée par l'auteur.

25. The 42nd Parallel, "Camera Eye 3", p. 37.

26. J.D. Brantley, 1968: 76 .

27. Nineteen Nineteen, "Camera Eye 34 », p. 480.

28. The Big Money, "Camera Eye 50 », p. 1106.

29. Ibid.: 1105.

30. Ibid.: 1106

31. Ibid., "Camera Eye 51 ", p. 1153.

32. Ibid., "Camera Eye 44», p. 755.

\section{RÉFÉRENCES BIBLIOGRAPHIQUES}

BALAT, M. [1994] : "Sur la division du sujet", S-Revue européenne de sémiotique, vol. 6 (3-4), 691-733.

Brantley, J. D. [1968]: The Fiction of John Dos Passos, The Hague/Paris, Mouton.

DeLEDALLE, G. [1979]: Théorie et pratique du signe. Introduction à la sémiotique de Charles S. Peirce, avec la collaboration de J. Réthoré, Paris, Payot.

Dos PAssos, J. [1966] : U.S.A., London, New York, Victoria, Toronto, Auchland, Penguin Books, 1 ère publication de la trilogie aux U.S.A.,

1938. Publication de chacun des volumes de la trilogie: The 42nd

Parallel, 1930; Nineteen Nineteen, 1932; The Big Money, 1936.

PeirCE, C. S. [1931-35, 1958]: Collected Papers, vol. I-VI (sous la dir. de

C. Hartshorne \& P. Weiss) et vol. VII-VIII (sous la dir. de A.W. Burks),
Cambridge, Mass., The Belknap Press.

PHILPS, D. [1997]: «À la Recherche du sens perdu: 〈sn>, du marqueur au mythe", Anglophonia, Sigma n², Université de Toulouse Le Mirail, Presses Universitaires du Mirail, 209-238.

RÉTHORÉ, J. [1998a] : «L'Interprétation, fondement du langage et condition de toute signification", dans Protée, vol.26, nº 1

( Interprétation», sous la dir. de L. Hébert), 19-27;

[1998b]: «L'entour du signe écrit: énonciation et lecture. De la nécessaire division du sujet ", Degrés, (sous la dir. de Helbo), Bruxelles, à paraître;

[1989]: «Sémiotique et langues naturelles", dans Peircean Semiotics in Perpignan (sous la dir. de G. Deledalle), Vienne, S-European Journal for Semiotic Studies, vol. 1-4, 611-630;

[1988]: «La Proposition chez Peirce: sujet-prédicat. Et la copule?», dans Fondements de la sémiotique (sous la dir. de G. Deledalle), Degrés, n $54-55$, Bruxelles, e1-e11.

Ruhlen, M. [1997]: L'Origine des langues, Paris, Éd. Belin pour la traduction française. Titre original: The Origin of Language. Tracing the Evolution of the Mother Tongue, New York, John Wiley \& Sons, 1994. SAINT-John Perse [1970-1995]: Amers, suivi de Oiseaux, Paris, Gallimard, coll. «Poésie/Gallimard».

StENGERS, I. [1997] : Au Nom de la flèche du temps: le défi de Prigogine, Cosmopolitiques V, Paris, La Découverte/Les Empêcheurs de penser en rond.

TESNIÈRE, L. [1959]: Éléments de syntaxe structurale, Paris, Klincksieck, 2eéd. revue et corrigée, $3^{e}$ tirage 1976. 